\title{
The Flow and Adsorption of DNA Polymers Near Surfaces
}

\author{
Ronald G. Larson, Lei $\mathrm{Li}^{1}$, Manish Chopra ${ }^{2}$, and Mark A. Burns \\ Department of Chemical Engineering, University of Michigan, Ann Arbor, MI 48109-2136, U.S.A. \\ ${ }^{l}$ Current address: Department of Pharmaceutical Sciences, University of Michigan, Ann Arbor, MI. \\ ${ }^{2}$ Current address: Mckinsey \& Company, Detroit, MI.
}

\begin{abstract}
We discuss how agreement between single-molecule imaging methods applied to DNA molecules in flow [1,2] and Brownian dynamics simulations using bead-spring or bead-rod course-grained models [3,4] can be extended to study the interactions of flowing DNA polymers with surfaces, which are of importance in the development of microfluidic devices for processing of DNA and other large molecules for genomics, bio-assays, combinatorial polymer science, etc. Using single-molecule experiments and Brownian dynamics simulations we review work on isolated DNA molecules near adsorbing and non-adsorbing walls in the presence of a simple shearing flow and in an evaporating droplet. The former flow is predicted to produce highly stretched adsorbed molecules due to the prevalence of end-sticking, following by regular unraveling from one end to the other and laying down of the molecule onto the surface. In the drying-droplet flow, this process is inhibited by the downward convection, which drives the molecule towards the surface, resulting in complete adhesion before unraveling is complete. Experimental studies using surfaces treated with APTES (3-aminopropyltriethoxysilane) to produce strong sticking of DNA confirm the Brownian dynamics predictions for the drying flow containing DNA. In simple shearing flow, an unusual, and unexplained, interaction of DNA with the surface inhibits stretching, at distances as great as 20 microns from the surface.
\end{abstract}

\section{INTRODUCTION}

Recent advances in single-molecule imaging methods applied to DNA molecules in flow [1,2] and advances in computer speed have allowed detailed comparisons to be made between observed and predicted behavior of dilute polymeric DNA molecules in simple flows. These have shown that the conformations and rheology of DNA molecules in bulk solution can be predicted with high accuracy by Brownian dynamics simulations using bead-spring or bead-rod course-grained models that include hydrodynamic interactions only in the choice of the effective bead drag coefficient $[3,4]$. We have shown that more complex effects of deformation-dependent hydrodynamic interactions are expected to be important in synthetic polymers such as high-molecular-weight polystyrene in dilute solution, but that these effects are negligible in DNA due to its open configuration at rest, owing to its large persistence length [5].

A logical next step is to extend these methods to complex flows, and to the interactions of flowing DNA polymers with surfaces, which are of importance in the application of microfluidic devices to the processing of
DNA and other large molecules for genomics, bioassays, combinatorial polymer science, etc. Using single-molecule experiments and Brownian dynamics simulations we have considered isolated DNA molecules near adsorbing and non-adsorbing walls in the presence of a simple shearing flow and in an evaporating droplet. We review this work here.

\section{FLOW FIELDS}

In simple shearing flow, Brownian dynamics simulations in the absence of hydrodynamic interactions predict that the molecules will become highly stretched as they become adsorbed irreversibly onto a surface [6]. This occurs becomes of endsticking, i.e., the tendency of the molecule to adsorb first at its end, and the to unravel onto the surface in the shearing flow. The end sticking prevents the molecule from undergoing continual tumbling and collapse, as well as stretching, in bulk shearing flow. The result is that the molecule, once anchored to the surface, can 
become highly stretched in the shearing flow, before it becomes irreversibly adsorbed onto the surface.

To extend these results to a complex flow, we need to solve for the flow field of a complex flow, and use this flow in a Brownian dynamics simulation to predict molecular stretch during DNA deposition. As a test problem, we have chosen the flow in a drying water droplet resting on a substrate. Because of the pinned contact line, the droplet does not shrink its radius until the very last stages of drying, but instead shrinks its height. As a result, fluid that evaporates from the edge of the droplet must be replaced by fluid flowing to the edge from the droplet center [7]; see Fig. 1.

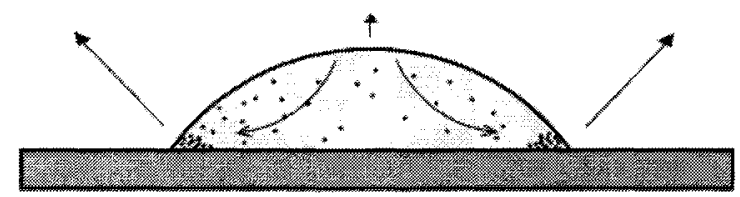

Figure 1. The flow in a drying droplet with pinned contact line.

This flow convects solute towards the droplet edge, where it deposits it in a ring [7], or "water spot," frequently seen on dishware that has been left to dry.

Besides being an annoyance, the "coffee ring" effect can be used to advantage in genomics applications. Schwartz and co-workers [8] have shown that the flow in a drying droplet can be used to stretch out and deposit DNA molecules, whereupon they can be subjected to a restriction enzyme digestion, and the length and relative positions of the fragments measured by simple fluorescence optical microscopy, used DNA stained by intercalating dyes.

The droplet drying flow, then, is an attractive one in which to carry out Brownian dynamics analyses of DNA stretch and deposition. Since the flow is not only multi-dimensional, but also time dependent, to carry out Brownian dynamics simulations, one would need either an analytic solution to the droplet-drying flow, or a numerical solution for this flow that is carried out simultaneously with the Brownian dynamics simulations. We have pursued the former approach, but used a numerical solution to check the accuracy of our analytic solution.

To obtain an analytic solution, we start with the approximate solution of Deegan et al. [7] for the heightaveraged radial velocity field, which is

$$
\overline{\mathrm{u}}_{\mathrm{r}}=\frac{1}{4} \frac{1}{\mathrm{t}_{\mathrm{f}}-\mathrm{t}} \frac{\mathrm{R}}{\tilde{\mathrm{r}}}\left[\left(1-\tilde{\mathrm{r}}^{2}\right)^{-\lambda(\theta)}-\left(1-\tilde{\mathrm{r}}^{2}\right)\right]
$$

Here, $\lambda$ is a parameter reflecting the uniformity of evaporation, which is 0.5 for a flat droplet. The accuracy of this expression has been thoroughly explored in a recent experimental and finite-element study of droplet evaporation by $\mathrm{Hu}$ and Larson [9], who found that for small droplets whose shape is that of a spherical cap resting on a surface with contact angle $\theta$, the above expression for evaporative flux is accurate, with $\lambda=\frac{1}{2}-\frac{\theta}{\pi}$, where $\theta$ is the contact angle in radians. From the approximate evaporative flux expression, the entire axisymmetric flow field inside the droplet, including both radial and axial components, can be obtained by using the lubrication approximation and the zero-shear-stress boundary condition on the free airliquid surface and the no-slip boundary condition on the glass substrate. The zero-shear-stress boundary condition neglects Marangoni flow produced by temperature gradients that arise due to evaporative cooling. However, for water, thermal Marangoni flows are generally not observed, and so these surfacetension-gradient effects can be neglected and the noshear-stress boundary condition on the free surface can be used. With these boundary conditions and the continuity equation, the radial and axial velocity components in dimensionless form can be given as [6]:

$$
\begin{aligned}
& \tilde{\mathrm{u}}_{\mathrm{r}}=\frac{3}{8} \frac{1}{1-\tilde{\mathrm{t}}} \frac{1}{\tilde{\mathrm{r}}}\left[\left(1-\tilde{\mathrm{r}}^{2}\right)-\left(1-\tilde{\mathrm{r}}^{2}\right)^{-\lambda(\theta)}\right]\left\{\frac{\tilde{\mathrm{z}}^{2}}{\tilde{\mathrm{h}}^{2}}-2 \frac{\tilde{\mathrm{z}}}{\tilde{\mathrm{h}}}\right\} \\
& \tilde{\mathrm{u}}_{2}=\frac{3}{4} \frac{1}{1-\tilde{\mathrm{t}}}\left[1+\lambda(\theta)\left(1-\tilde{\mathrm{r}}^{2}\right)^{-\lambda(\theta)-1]}\right]\left\{\frac{\tilde{\mathrm{z}}^{3}}{3 \tilde{\mathrm{h}}^{2}}-\frac{\tilde{\mathrm{z}}^{2}}{\tilde{\mathrm{h}}\}}+\right. \\
& \frac{3}{2} \frac{1}{1-\tilde{\mathrm{t}}}\left[\left(1-\tilde{\mathrm{r}}^{2}\right)-\left(1-\tilde{\mathrm{r}}^{2}\right)^{-\lambda(\theta)}\right]\left\{\frac{\tilde{\mathrm{z}}^{2}}{2 \tilde{\mathrm{h}}^{2}}-\frac{\tilde{\mathrm{z}}^{3}}{3 \tilde{\mathrm{h}}^{3}}\right\} \tilde{\mathrm{h}}(0, \tilde{\mathrm{t}})
\end{aligned}
$$

Here the dimensionless variables are defined as follows:

$$
\begin{aligned}
& \tilde{\mathrm{u}}_{\mathrm{r}}=\frac{\mathrm{u}_{\mathrm{r}} \mathrm{t}_{\mathrm{f}}}{\mathrm{R}}, \quad \tilde{\mathrm{u}}_{\mathrm{z}}=\frac{\mathrm{u}_{\mathrm{z}} \mathrm{t}_{\mathrm{f}}}{\mathrm{h}_{0}}, \quad \tilde{\mathrm{t}}=\frac{\mathrm{t}}{\mathrm{t}_{\mathrm{f}}}, \quad \tilde{\mathrm{r}}=\frac{\mathrm{r}}{\mathrm{R}} \\
& \tilde{\mathrm{z}}=\frac{\mathrm{z}}{\mathrm{h}_{0}}, \tilde{\mathrm{h}}=\frac{\mathrm{h}}{\mathrm{h}_{0}}
\end{aligned}
$$

and $u_{r}$ and $u_{z}$ are the dimensional radial and axial velocity components respectively. $R$ is the radius of the droplet, which is assumed to be constant with time and $r$ is the radial position. $t_{f}$ is the total drying time, $t$ is the time, $h_{0}$ is the initial height of the droplet, $h$ is the instantaneous height at time $t$ at a radial position $r, \theta$ is the instantaneous contact angle and is a linearly decreasing function of time. A key feature of this solution is that the velocities become singular at the end of drying. 
This flow field was tested by using a particletracking velocimetry method, described elsewhere and found to be reasonably accurate [10]. We therefore combined this flow field with Brownian dynamics simulations of lambda-phage DNA molecules, to predict their deformation and adhesion to a sticky glass substrate during the droplet-drying flow [11]. Irreversible DNA adhesion was incorporated into the Brownian dynamics simulations by freezing onto the glass substrate any bead of the bead-spring chain that contacted the surface during the simulations. Effects of "lubrication forces" or local electrostatic interactions with the substrate were neglected.

\section{BROWNIAN DYNAMICS SIMULATIONS}

Brownian dynamics simulations of a lambda-phage DNA molecule were carried out using the well-known bead-spring model, using methods described elsewhere [6,11]. The parameters of the bead-spring model, including bead drag coefficient and elastic spring constants, are chosen a priori using the known molecular properties of lambda-phage DNA [3]. Interactions between one molecule and another are neglected, since we are focusing on dilute solutions with low surface coverage. We ignore hydrodynamic lubrication forces of the thin fluid layers between the polymer and the substrate, and hydrodynamic interactions between the DNA and the wall. We also neglect monomer-monomer excluded-volume interactions within a single molecule. We analyze 2,000 chains at each evaporation rate (drying time), distributed initially uniformly throughout the volume of the droplet. We store the maximum stretch length (defined as the distance between two farthest-separated segments) of the molecule at the moment of "touchdown" of each bead until the molecule adsorbs fully (i.e., all the beads have been bound to the surface) [11]. We validated the accuracy of the simulations by varying the number of beads and the time step, and found the simulations to be quantitatively robust to variations in these parameters.

The simulations show that, in the drying-droplet flow, the process by which the chain unravels onto the surface, seen in simulations of the simple shear flow, is inhibited by the downward convection, which drives the molecule towards the surface, resulting in complete adhesion before unraveling is complete [11]. Thus, in the simulations, the molecular stretch is highly heterogeneous, and on average, rather weak.

\section{EXPERIMENTS}

As described in more detail elsewhere [11], we carried out experimental studies of DNA deposition from a drying droplet using glass surfaces treated with APTES (3-aminopropyltriethoxysilane) to produce a positively charged surface, and therefore strong sticking of negatively charged DNA molecules. Specifically, $0.5 \mu \mathrm{l}$ droplets of $50 \mathrm{pg} / \mathrm{ml}$ DNA solutions were deposited onto the APTES-coated glass coverslips. The droplet was shielded from air currents using a cylindrical cap (6 $\mathrm{mm}$ in diameter and $6 \mathrm{~mm}$ in height) with an open top to permit evaporation. The evaporation rate could be controlled using a Millipore membrane on top of the droplet chamber. We used $10 \mathrm{mM}$ Tris- $\mathrm{HCl}$ and $1 \mathrm{mM}$ EDTA at $\mathrm{pH} 8.0$ as buffer solutions, with $50 \mathrm{pg} / \mathrm{ml} 48.5$-kbp $\lambda$-phage DNA (New England BioLab) stained with YOYO-1 fluorescent dye (Molecular Probe Inc.) at a dye-to-base-pair molar ratio of $1: 8$, and $10 \% \beta$-mercaptoethanol (Sigma). We note that we use a higher concentration of $\beta$ mercaptoethanol than the $4 \%$ used in Smith and Chu's experiments (1999) because the former yields greater delaying of photo-bleaching. The effect of this on the DNA molecular persistence length is here neglected. After the droplet had dried out, $10 \mathrm{ml}$ of $10 \mathrm{mM}$ Tris$\mathrm{HCl}$ and $1 \mathrm{mM}$ EDTA buffer solution with $10 \% \quad \beta$ mercaptoethanol were deposited onto the dried spot to re-wet the surface for observation of the DNA. Stained DNA molecules, either in bulk solution or on the coated glass slips, were visualized using a Nikon TE200 fluorescent microscope with a $100 x$ objective, with a digital interline CCD camera MicroMax 1300YHS (Princeton Instruments, distributed by Fryer Co.) to capture both still and dynamic images at a resolution of $1300 \times 1030$ using full-chip acquisition. The image acquisition software MetaView/MetaMorph version 4.5 (Universal Image, distributed by Fryer Co.) was used to control the camera, the XYZ stage motor (Prior Inc.), and the electronic shutter (Uniblitz VMM-D1, Vincent Associates), and to run the image-analysis software.

These experiments confirm the Brownian dynamics predictions for the drying flow containing DNA. We showed that the combination of a continuum fluid dynamical solution for the flow in an evaporating droplet with Brownian dynamics simulations of DNA molecules in that flow, are able accurately to predict the stretching and deposition of DNA molecules onto a substrate during droplet drying; see Fig. 2. The average stretch of the deposited lambda DNA molecules is only around $15 \%$ of full stretch, even under rather rapid evaporation conditions in which a $1-\mathrm{mm}$ radius droplet dries out in three minutes. The distribution of stretch is well predicted, as is the distribution of orientation angles with respect to the flow direction [11]. 

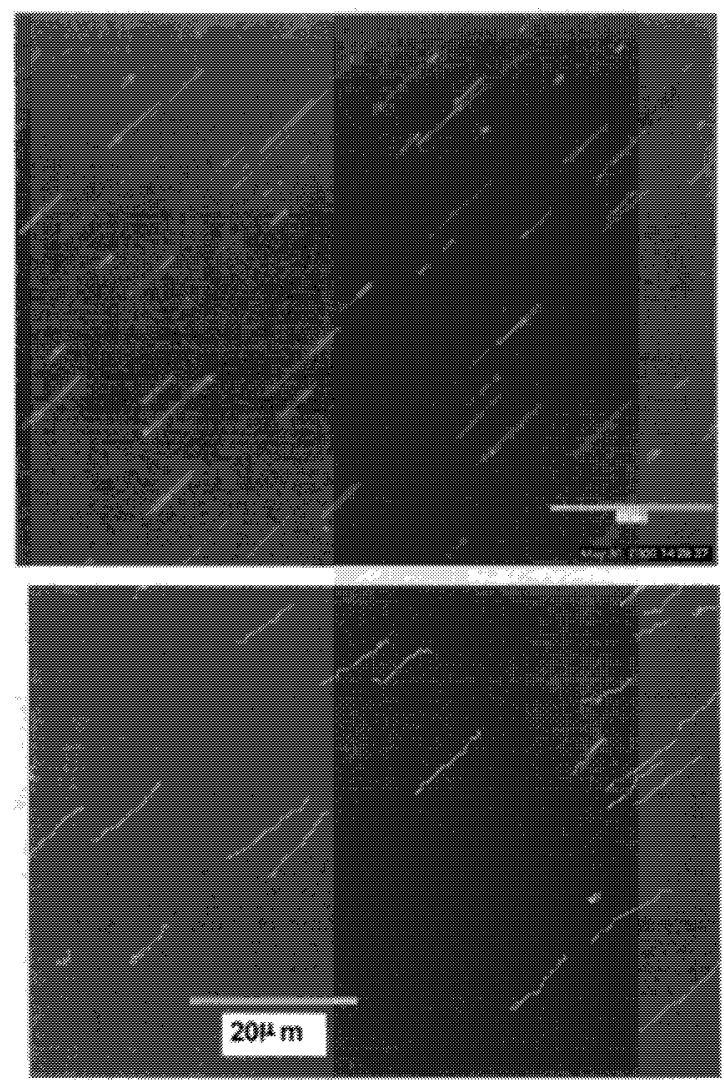

Figure 2. DNA images at the edge of a droplet (radial position $>90 \%$ of the droplet radius) at 3 mins. drying time, $20 \mu \mathrm{m}$ from the edge of the droplet. The top is an experimental image and the bottom is the simulated one, under conditions identical to those in the experiments. Histograms showing distribution of stretch and orientation from the simulations are in good agreement with those measured. The scale bar represents $20 \mu \mathrm{m}$ in length. (The figure is taken from ref. 11 , used with permission, from the Journal of Rheology).
We also carried out experiments in simple shearing flows, using a torsional shearing apparatus, consisting of a pair of parallel glass disks, the upper of which could be rotated using a motor, and the lower of which was the cover-slip for an inverted fluorescence microscope. In this flow, we also observed DNA stretch, not only after deposition onto an APTES-treated glass coverslip, but also in the fluid undergoing shear, above the cover slip. We observed that, both after adsorption on the coverslip, and in the fluid near the coverslip, the DNA was much less stretched than predicted by theory [12]. At this point, we do not have an explanation for this unusual behavior.

\section{REFERENCES}

1. Perkins, T.T., Smith, D.E., and Chu, S., Science, 276, 2016-2021 (1997).

2. Smith, D.E., and Chu, S. Science 281, 1335-1340 (1998).

3. Larson, R.G., Hu, H. Smith, D.E., and Chu, S., J. Rheol., 43 267-304 (1999).

4. Hur, J.S., Shaqfeh, E.S.G.S., and Larson, R.G., J. Rheol. 44 713-742 (2000).

5. Hsieh, C.-C., and R.G. Larson, J.Non-Newt. Fluid Mech.,in press (2003).

6. Chopra, M. and Larson, R.G., J. Rheol., 46, 831-862, (2002).

7. Deegan, R. D., Bakajin, O., Dupont, T. F., Huber, G., Nagel, S. R., Witten, T. A., Nature 389, 827-829 (1997).

8. Jing J., Reed, J., Huang, J., Hu, X., Clarke, C. V., Edington, J., Housman, D., Anantharaman, T. S., Huff, E.J., Mishra, B., Porter, B., Shenker, A., Wolfson, E., Hiort, C., Kantor, R., Aston, C., Schwartz, D. C., Proc. Natl. Acad. Sci. USA 95, 8046-8051 (1998).

9. Hu, H. and Larson, R.G., J. Phys. Chem. 106, 1334-1344 (2002).

10. Hu, H. and Larson, R.G., Micro-Fluid Dynamics in an Evaporating Sessile Droplet, to be published.

11. Chopra, M., Li, L., Burns, M.A., and Larson, R.G., J. Rheol., in press, (2003).

12. Lei, L., Hu, H., and Larson, R.G., Rheol Acta, submitted (2003). 